\title{
GLOBAL ESTIMATION OF SOIL MOISTURE PERSISTENCE WITH L AND C-BAND MICROWAVE SENSORS
}

\author{
M. Piles ${ }^{1}$, R. van der Schalie ${ }^{2}$, A. Gruber ${ }^{3}$, J. Muñoz-Marí ${ }^{1}$, G. Camps-Valls ${ }^{1}$ \\ A. Mateo-Sanchis ${ }^{1}$, W. Dorigo ${ }^{4}$, R. de Jeu ${ }^{2}$ \\ ${ }^{1}$ Image Processing Lab (IPL), Universitat de València, València, Spain \\ ${ }^{2}$ VanderSat B.V., Wilhelminastraat 43A, 2011 VK Haarlem, Netherlands \\ ${ }^{3}$ Department of Earth and Environmental Sciences, KU Leuven, Heverlee, B-3001, Belgium \\ ${ }^{4}$ Department of Geodesy and Geo-Information, Vienna University of Technology, 1040 Vienna, Austria
}

\begin{abstract}
Measurements of soil moisture are needed for a better global understanding of the land surface-climate feedbacks at both the local and the global scale. Satellite sensors operating in the low frequency microwave spectrum (from 1 to 10 $\mathrm{GHz}$ ) have proven to be suitable for soil moisture retrievals. These sensors now cover nearly 4 decades thus allowing for global multi-mission climate data records. In this paper, we assess the possibility of using L-band (SMOS) and C-band (AMSR2, ASCAT) remotely sensed soil moisture time series for the global estimation of soil moisture persistence. A multi-output Gaussian process regression model is applied to ensure spatio-temporal coverage of the satellite data sets. It allows a robust computation of temporal autocorrelation and e-folding times. Results over a selection of catchments reveals general agreement between the response of in-situ and satellite microwave observations to hydrological processes. The response of the uppermost-modeled soil moisture layer of GLDAS-1-Noah agrees well with that of the observations, whereas major differences are displayed by MERRA2 reanalysis. The temporal dynamics of the three microwave sensors are shown to be consistent, close to in-situ and to GLDAS-1-Noah, which supports their combination for the global estimation soil moisture persistence.
\end{abstract}

Index Terms - Soil moisture, microwave radiometry, temporal autocorrelation, SMOS, AMSR2, ASCAT, Gaussian processes (GPs), regression, gap-filling

\section{INTRODUCTION}

During the last decade, the interest in the protected microwave L-band and technological advances in instrumentation and space technology led to the two first space missions dedicated to measuring soil moisture: ESA's Soil Moisture and Ocean Salinity (SMOS) and NASA's Soil Moisture Active

This research has been conducted within a Visiting Scientist Activity under the ESA CCI Soil Moisture Project, Phase 2. GCV was funded by the European Research Council (ERC) under the ERC-CoG-2014 SEDAL project (grant agreement 647423).
Passive (SMAP). Also, microwave satellite sensors operating at higher frequencies with long technological heritage, e.g. C-band ASCAT and AMSR2, that were initially devoted to atmospheric and/or oceanic sensing have been proved suitable for soil moisture retrieval. In comparison to L-band, Cband sensors have a shallower soil sensing depth (top $1 \mathrm{~cm}$ vs. $5 \mathrm{~cm}$ ) and a larger attenuation in presence of vegetation. Nonetheless, higher frequency missions can complement recent L-band missions and allow for multi-decadal soil moisture observational data records partially covering the last 38 years (e.g. ESA CCI Soil Moisture [1]).

In this work, an autocorrelation analysis is presented to capture the general temporal dynamics of soil moisture data products from three satellites (SMOS, AMSR2, ASCAT), and two state-of-the-art reanalysis (GLDAS-1-Noah, MERRA2). Given the large gap rates, we propose a multi-output Gaussian Process (GP) regression model to ensure spatio-temporal coverage of the satellite data sets [2]. The method implicitly performs an information transfer across the three satellite time series. It allows a robust computation of temporal autocorrelation and e-folding times, as a measure of soil moisture persistence. The study period covers six years, starting in June 2010. The analysis is performed over a selection of in-situ catchments from the International Soil Moisture Network (https://ismn.geo.tuwien.ac.at). Based on the skills and differences found between the different soil moisture products a methodology for the global estimation of soil moisture persistence is proposed. It uses $\mathrm{L}$ and $\mathrm{C}$-band satellite microwave observations and deals with the natural presence of observational gaps in the time series.

The paper is organized as follows. In Section 2 we briefly introduce the proposed methodological approach. Section 3 presents the experimental results, and we end up the work in Section 4 with some remarks and future work.

\section{PROPOSED APPROACH}

We characterized the soil moisture persistence or "soil memory" in terms of temporal autocorrelation (lagged correlation) of the different data records. Although modeled, satellite and 
in-situ observed soil moisture products capture processes occurring at different spatial scales, therefore making their comparison challenging, they should generally agree in terms of temporal dynamics (e.g. trend) and have a similar response to rainfall if the rainfall was equally distributed [3].

The autocorrelation analysis is a powerful tool to describe the direct soil moisture response to hydrological processes $[4,5]$. Yet, a major obstacle for its applicability to observational time series is the presence of missing data that prevents a robust computation in many regions of the planet. In this study, a multi-output GP regression technique [2] is applied to the satellite data to ensure enough spatio-temporal coverage of the data sets in snow-free continental areas. The method takes into account the relationships among SMOS, ASCAT and AMSR2 soil moisture time series and builds a cross-domain kernel function able to transfer information between time series. The learned relations are exploited to do inferences on regions where no data (gaps) are available and provides a reconstructed prediction for each data set.

Typically, the temporal autocorrelation is applied to anomaly time series to prevent correlations of the time series with the seasonal cycle. Here, a stable seasonal component at the daily scale has been obtained from monthly means, using cubic splines. Soil moisture persistence is then characterized by the autocorrelation curve and the characteristic time lag or e-folding time (the time at which the autocorrelation drops to $1 / e)$ [4].

The robustness of the approach is evaluated at three insitu soil moisture networks: REMEDHUS in Spain (17 stations [6]), HOBE in Denmark (31 stations [7]) and DAHRA in Senegal (1 station [8]). In terms of temporal coverage, they are representative of best-case (REMEDHUS), averagecase (HOBE) and wort-case (DAHRA) scenarios, with SMOS providing a coverage during the study period of 96,65 and $45 \%$, respectively. Autocorrelation curves and characteristic lags have been obtained for in-situ, satellite (original and reconstructed) and uppermost-modeled soil moisture layers of GLDAS and MERRA reanalysis.

\section{RESULTS}

\subsection{Gap filling with multi-output Gaussian Processes}

The spatio-temporal coverage of microwave observations for the study period is shown in Fig.1. It shows that combining the three satellite observations most continental pixels have more than $80 \%$ temporal coverage, whereas with SMOS alone the mean coverage is about $50 \%$ (Fig. 1a). Not surprisingly, coverage is still limited at high latitudes (due to snow masking microwave observations) and areas of dense tropical forests and complex topography where soil moisture retrievals have a high uncertainty (Fig 1b).

A multi-output GP regression is applied here to combine the complementary information captured by the three microwave sensors and provide continuous time series for a robust assessment of soil moisture persistence metrics. The
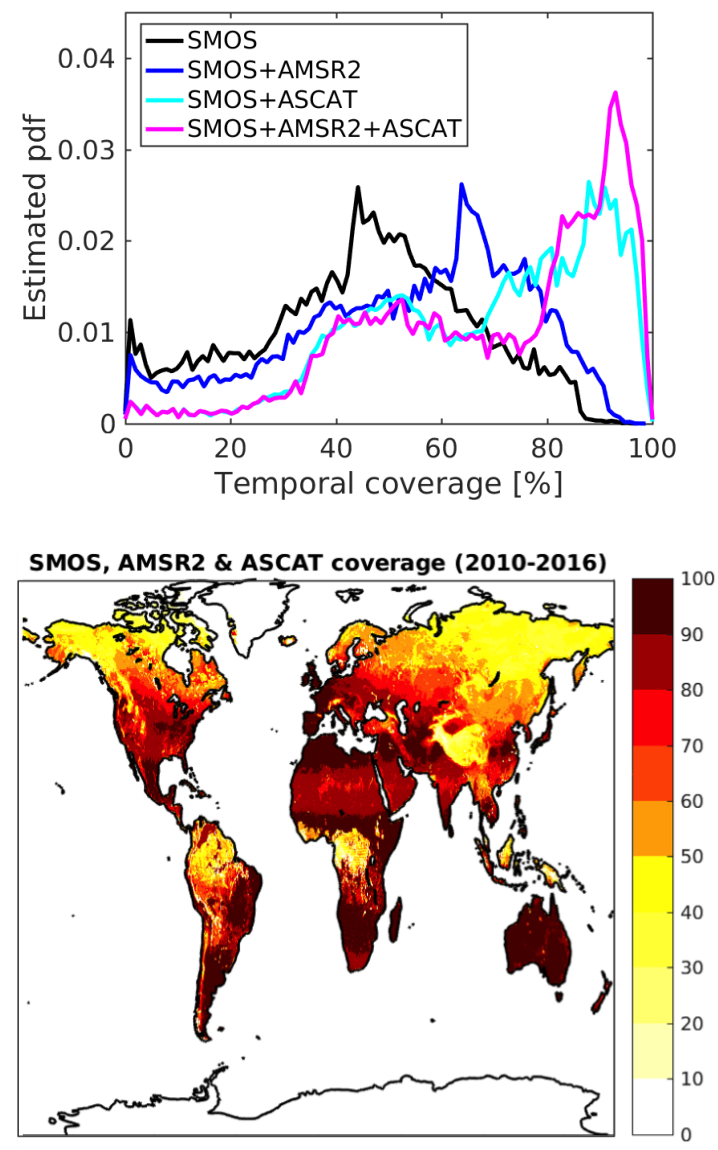

Fig. 1. Temporal coverage for the 6-year the study period [\%]. Top: probability density function of temporal coverage considering all continental pixels, for different combinations of satellite data. Bottom: map of temporal coverage resulting from the combination of SMOS, AMSR2 and ASCAT data.

interested reader can find details on this particular model in $[2,9]$, and more details will be given at the time of the conference. Results of the application of the proposed GP over REMEDHUS, HOBE and DAHRA networks are shown in Fig. 2, together with the original satellite time series and the in-situ data as a benchmark. For REMEDHUS and HOBE, it can be seen that reconstructed soil moisture time series follow closely the original time series, capturing the wetting-up and drying-down events and filling the missing information (e.g. see in HOBE the dry-down in February 2014 which was captured only by AMSR 2 and is reproduced by the three reconstructed time series). Pearson correlation and root-mean-squared errors with respect to in-situ data in these two networks are not affected by the reconstruction. The reconstructed signals at DAHRA, however, are too smooth and not representative (high frequency changes are lost). This is due to the fact that, in this region, satellites barely overlap and the combined coverage is very poor, probably due to its proximity to the coast. These results suggest that a minimum 

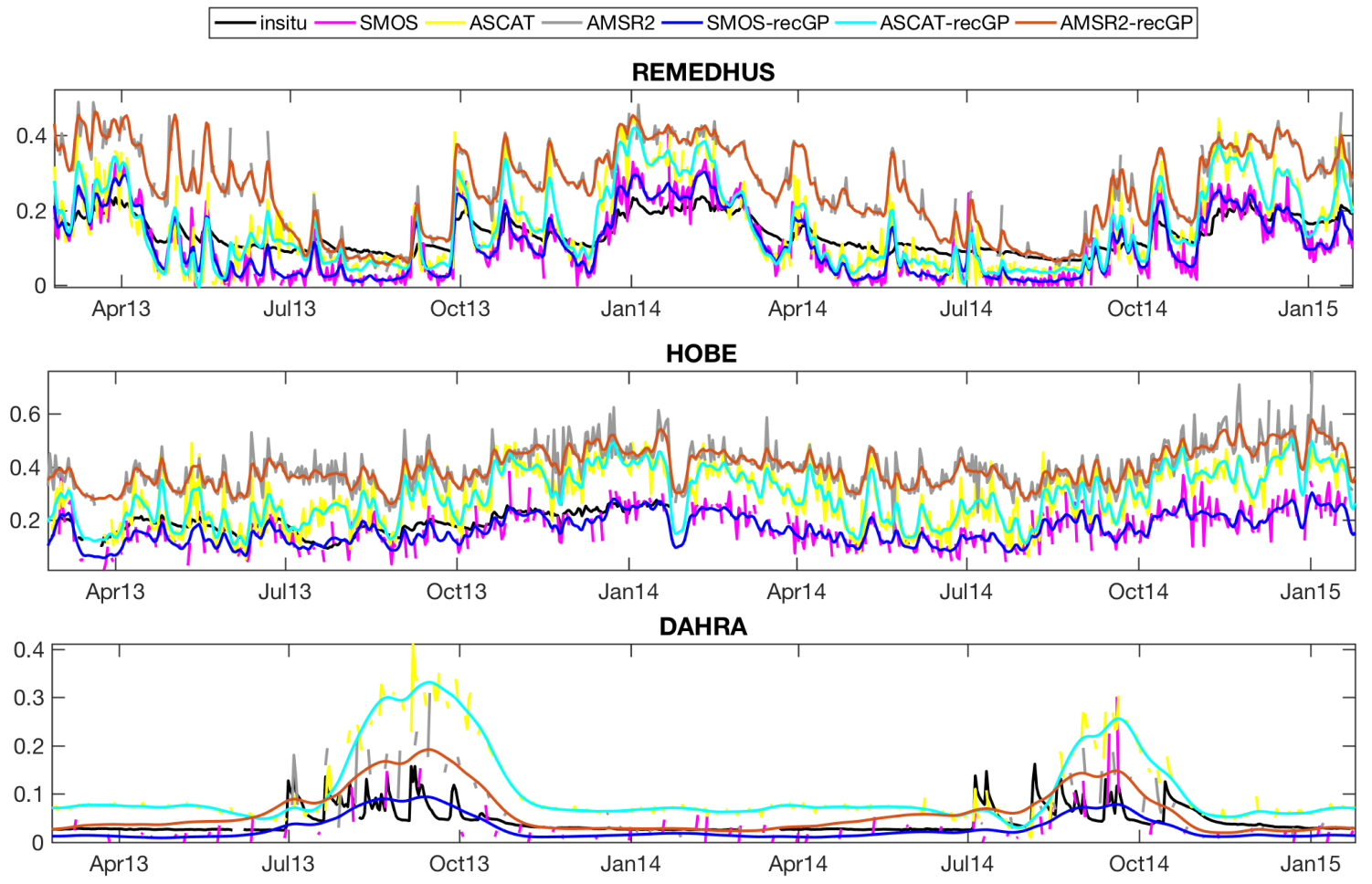

Fig. 2. Time series of in-situ, satellite-based (SMOS, ASCAT, AMSR2), and reconstructed soil moisture [m $\left.\mathrm{m}^{3} \mathrm{~m}^{-3}\right]$ at the REMEDHUS catchment during the 6-year study period. Reconstructed satellite time series obtained from the multi-output Gaussian Process regression are also shown.

number of combined observations (around 50-60\% of temporal coverage) is required to provide consistent predictions using the proposed GP model.

\subsection{Temporal autocorrelation}

Autocorrelation graphs of the different soil moisture data records over the REMEDHUS and HOBE networks are shown in Fig. 3. It illustrates that, in these sites, the temporal response of the uppermost-modeled soil moisture layers to hydrological processes is generally different for GLDAS and MERRA, with GLDAS showing a characteristic curve much closer to both in-situ and satellite observations. Comparing satellite estimates, it can be seen that ASCAT and AMSR2 generally display lower persistence than SMOS (lower autocorrelation values for most time lags). This is in agreement with the theory, since a shallower layer of the soil contributes to surface emissivity at C-band than at L-band (1 cm vs. 5 $\mathrm{cm})$ and therefore should lead to lower memory. The distinct properties shown by AMSR2 and ASCAT can (in part) be due to their operating mode (passive vs. active sensing). Despite some differences, it is remarkable that the temporal response of the three satellite data sets are consistent.

It should be noted that autocorrelation estimates are unreliable when the data has a high number of missing values (as a rule of thumb, when more that $1 / 3$ of data is missing). This is the case of AMSR2 in REMEDHUS and the three satellite time series in HOBE, as evidenced in their noisy autocorrelations (see Fig.3). Characteristic curves of reconstructed satellite time series are similar to the one of in-situ, with e-folding times of 9-11 days over REMEDHUS vs. 16 for in-situ and 6-8 days over HOBE vs. 10 for in-situ. GLDAS e-folding times lie between satellite and in-situ values (15 for REMEDHUS, 10 for HOBE). MERRA, in turn, results in unrealistic efolding times (30 days for REMEDHUS and 164 for HOBE).

\section{DISCUSSION AND CONCLUSION}

An autocorrelation analysis has been performed to characterize the soil moisture temporal dynamics displayed by insitu measurements, satellite microwave observations (SMOS, AMSR2, ASCAT), and state of the art reanalysis (GLDAS-1Noah and MERRA2). A multi-output GP regression has been applied to intrinsically exploit the relationships among the three microwave sensors and deal with the natural presence of missing data in observational time series. Results have been presented over three catchments: REMEDHUS, HOBE and DAHRA, with a satellite coverage during the study period of 96, 65 and 45\%, respectively.

Reconstructed time series using the proposed GP model allow the analysis of autocorrelation curves and e-folding times over the REMEDHUS and HOBE catchments. Cov- 

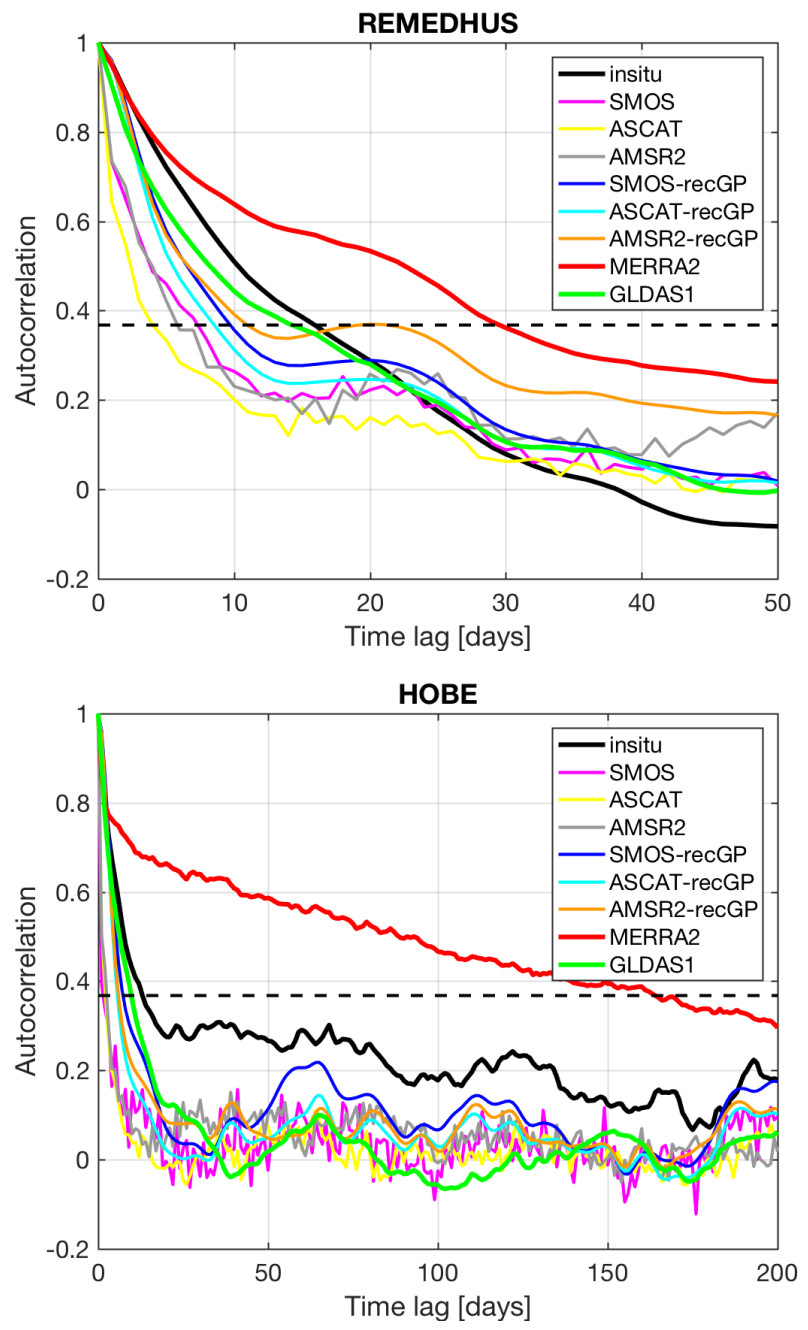

Fig. 3. Autocorrelation graphs of SMOS, AMSR2, ASCAT, GLDAS-1-Noah, MERRA2 and in-situ (average of available stations) soil moisture anomalies over REMEDHUS (top) and HOBE (bottom). The black dashed horizontal line indicates where the autocorrelation equals $1 / e$, corresponding to the characteristic lag-time at the $\mathrm{x}$-axis.

erage over DAHRA is too limited to provide a robust reconstruction using GPs alone. Further work will be directed towards the combined use of autoregressive models and GPs to allow reconstruction in pixels with less than $50-60 \%$ coverage. Also, the use of Gaussian or exponential kernels for smoother/sharper approximations of the signals and its impact in the autocorrelation function needs to be evaluated.

Despite some differences, the temporal response of the three microwave sensors are consistent. Characteristic curves of reconstructed time series are similar to the one of in-situ with slightly lower e-folding times (9-11 days vs. 16 over REMEDHUS, 6-8 days vs. 10 over HOBE). GLDAS characteristic curve agrees well with that of satellite data and insitu, whereas MERRA displays unrealistic e-folding times.
This analysis should be extended to a higher number of catchments to ensure representativity of results. A dedicated study should be performed over irrigated regions, which are naturally captured by the satellite instruments but not included in the models. Results show that the proposed approach allows the estimation of global soil moisture persistence patterns captured by $\mathrm{L}$ and $\mathrm{C}$-band microwave sensors. Regional maps of e-folding times will be shown at the time of the conference to reveal the soil moisture persistence patterns captured by the microwave sensors and how they differ from the ones obtained with GLDAS and MERRA reanalysis.

\section{REFERENCES}

[1] W.A. Dorigo et al., "ESA CCI soil moisture for improved Earth system understanding: State-of-the art and future directions," Remote Sensing of Environment, vol. 203, pp. 185 - 215, 2017, Earth Observation of Essential Climate Variables.

[2] G. Camps-Valls et al., "A survey on gaussian processes for earth observation data analysis: A comprehensive investigation," IEEE Geoscience and Remote Sensing Magazine, , no. 6, pp. 58 - 78, June 2016.

[3] K.T. Rebel et al., "A global analysis of soil moisture derived from satellite observations and a land surface model," Hydrology and Earth System Sciences, vol. 16, no. 3, pp. 833-847, 2012.

[4] T.L. Delworth and S. Manabe, "The influence of potential evaporation on the variabilities of simulated soil wetness and climate," Journal of Climate, vol. 1, no. 5, pp. 523$547,1988$.

[5] J.K. Entin et al., "Temporal and spatial scales of observed soil moisture variations in the extratropics," Journal of Geophysical Research: Atmospheres, vol. 105, no. D9, pp. 11865-11877.

[6] N. Sanchez et al., "Validation of the SMOS L2 soil moisture data in the REMEDHUS network (Spain)," IEEE Transactions on Geoscience and Remote Sensing, vol. 50, no. 5, pp. 1602-1611, May 2012.

[7] S. Bircher et al., "A soil moisture and temperature network for smos validation in western denmark," Hydrology and Earth System Sciences, vol. 16, no. 5, pp. 14451463, 2012.

[8] T. Tagesson et al., "Ecosystem properties of semiarid savanna grassland in west africa and its relationship with environmental variability," Global Change Biology, vol. 21, no. 1, pp. 250-264.

[9] M. A. Álvarez, L. Rosasco, and N. D. Lawrence, "Kernels for vector-valued functions: A review," Found. Trends Mach. Learn., vol. 4, no. 3, pp. 195-266, Mar. 2012. 